\title{
Termografia Aplicada à Visualização de Transição de Camada Limite em Aerofólios
}

\section{Thermography Applied on Boundary Layer Transition Visualization in Airfoils}

\author{
Jorge H. Bidinotto ${ }^{1}$; Maximilian Kleinubing ${ }^{2}$; Fernando M. Catalano ${ }^{3}$; Eduardo M. Belo ${ }^{4}$
}

\begin{abstract}
Resumo
A indústria aeronáutica tem grande interesse no estudo dos fluxos aerodinâmicos laminares, uma vez que produzem força de arrasto reduzida, resultando em economia de combustível nas aeronaves. Esse tipo escoamento em torno de superfícies aerodinâmicas e sua transição a turbulento torna-se, portanto, importante para o projeto aeronáutico e validação de modelos de Dinâmica dos Fluidos Computacional (CFD). No entanto, tal determinação apresenta dificuldades experimentais, muitas vezes relacionadas com métodos invasivos, que podem influenciar a camada limite do escoamento na superfície, tornando os dados não-representativos. Este artigo apresenta uma metodologia para determinar a transição da camada limite de laminar a turbulento usando câmeras de imageamento infravermelho de alta sensibilidade. Este método baseia-se no fato de que uma diferença de temperatura na superfície do perfil aerodinâmico sob escoamento laminar e turbulento é induzida por uma diferença na troca de calor entre os dois regimes: a troca de calor é maior em escoamentos turbulentos e pode ser detectada por estas câmeras. A principal vantagem deste método está no fato de que é não-invasivo e, portanto, fornece dados experimentais mais consistentes a fim de validar modelos teóricos com melhor precisão. $\mathrm{O}$ trabalho baseia-se em uma apresentação teórica dos conceitos aplicados e ensaios em túnel de vento conduzidos no Laboratório de Aerodinâmica da Escola de Engenharia de São Carlos - USP, por membros desta instituição, em conjunto com pesquisadores da Bombardier Aviation e Universidade Federal de São Carlos - UFSCar.
\end{abstract}

Palavras-chave: Termografia. Camada Limite. Aerodinâmica. Imageamento Infravermelho.

\begin{abstract}
The Aeronautical industry has great interest in studying aerodynamic laminar flows, since they produce lower drag force, resulting in fuel economy on aircraft. This kind of flow determination around aerodynamic surfaces and its transition to turbulent becomes therefore of great interest for aeronautical design and Computational Fluid Dynamics model validation. However such determination presents experimental difficulties, often related with invasive methods, which can influence the flow and the surface boundary layer, making the data unrepresentative. This paper presents a methodology for determining the boundary layer transition from laminar to turbulent using high sensitivity infrared cameras. This method relies on the fact that a temperature difference on the airfoil surface under laminar and turbulent flow is induced by a difference in the heat exchange of two regimes. The heat exchange is higher in turbulent flows and can be detected by these cameras. Its main advantage lies in the fact that it is non-invasive and therefore provides more consistent experimental data, in order to validate theoretical
\end{abstract}

\footnotetext{
${ }^{1}$ Professor Doutor do Departamento de Engenharia Aeronáutica da Escola de Engenharia de São Carlos - USP; jhbidi@sc.usp.br.

${ }^{2}$ Flight Test Engineer, Bombardier Aviation; maxbing@gmail.com

${ }^{3}$ Professor Titular do Departamento de Engenharia Aeronáutica da Escola de Engenharia de São Carlos - USP; catalano@sc.usp.br

${ }^{4}$ Professor Titular do Departamento de Engenharia Aeronáutica da Escola de Engenharia de São Carlos - USP; belo@sc.usp.br
} 
models with better accuracy. The work is based on a theoretical presentation of applied concepts and wind tunnel tests conducted at the Laboratory of Aerodynamics of the Engineering School of São Carlos - USP, by members of this institution, together with researchers from Bombardier Aviation and Federal University of São Carlos - UFSCar.

Keywords: Thermography. Boundary Layer. Aerodynamics. Infrared Imaging.

\section{Introdução}

Durante as últimas décadas, várias aeronaves tiveram benefícios significativos de desempenho utilizando escoamento laminar sobre as suas superfícies para redução de arrasto aerodinâmico, melhorando seu desempenho e economizando combustível.

Escoamentos aerodinâmicos laminares podem ser definidos como aqueles em que as linhas de fluxo do fluido são paralelas e sem interrupção entre elas (ANDERSON, 1991).

A transição de escoamento de laminar a turbulento tem certas peculiaridades que afetam sua camada limite de várias formas, podendo apresentar alterações significativas na temperatura e portanto ser detectado por sensores térmicos, como as câmeras de imageamento infravermelho (MALERBA et al., 2008).

Esse tipo de camera apresenta uso nas mais variadas areas da tecnologia (HOLST, 2003; WYATT, 1987), como a área de vigilância ou a aeronáutica (McSHEA, 2010). Dentro desta última, destacam-se as aplicações em ensaios em voo (CLIFTON, 1996; FISHER; HORSTMANN; RIEDEL, 2003), aerodinâmica (BANKS, 2000; ZUCCHER et al., 2003) e conforto térmico (DEHNE; BOSBACH; HEIDER, 2012).
Este artigo apresenta uma proposta para a detecção de transição camada limite, testada em túneis de vento com aerofólios de baixa velocidade, usando câmeras de imageamento infravermelho. O texto apresenta alguns conceitos teóricos, a metodologia utilizada e os resultados desses testes. Também é apresentada uma proposta de possíveis dispositivos a serem utilizados em aeronaves reais e recomendações para estudos futuros.

\section{Teoria}

\section{Tecnologia Infravermelho}

Imagens infravermelho são geradas pelo processamento eletro-óptico da radiação emitida por qualquer objeto com o uso de equipamentos adequados chamados câmeras infravermelho.

Como radiação infravermelho entende-se a parte do espectro de radiações eletromagnéticas (Figura 1) com comprimento de onda entre 0,8 e $1000 \mu \mathrm{m}$, e se dividem em três categorias: radiação infravermelha curta $(0,8-1,5 \mu \mathrm{m})$, média $(1,5-5,6$ $\mu \mathrm{m})$ e longa (5,6-1.000 $\mu \mathrm{m})(\mathrm{HOLST}, 2003)$.

Figura 1: Espectro eletromagnético.

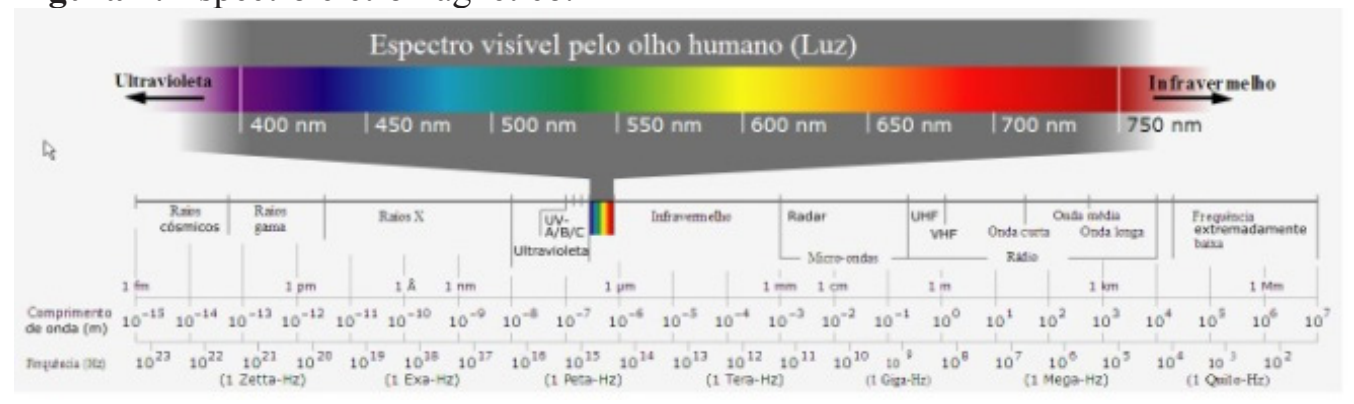

Fonte: PION, 2012. 
Aradiação térmica normalmente ocorre na região da radiação infravermelha média (para altas temperaturas) ou longa (para baixas temperaturas) e essa diferença de comprimento de onda (e consequentemente de frequência) é processada pela câmera infravermelho de forma a gerar as imagens baseadas em radiação. Algumas características importantes da radiação térmica são:

- Todos os objetos emitem radiação térmica;

- Ela não necessita de meio material para ocorrer;

- Atravessa facilmente a maioria dos gases;

- Dificilmente atravessa líquidos ou sólidos.

Além de emitir radiação, um objeto também absorve e reflete parte da radiação incidente do meio ambiente, ou permite que uma pequena parte desta radiação o atravesse (como através de uma lente). A lei de radiação total $(W)$ é derivada deste princípio físico, e pode ser estabelecida pela equação (1).

onde

$$
W=\alpha W+\tau W+\rho W
$$

$$
\alpha+\rho+\tau=1
$$

Os coeficientes $\alpha$, $\rho$, e $\tau$ descrevem a absorção de energia incidente do objeto $(\alpha)$, reflexão $(\rho)$ e transmissão $(\tau)$. O valor de cada coeficiente pode ser entre zero e um, dependendo da forma como o objeto absorve, reflete ou transmite a radiação Por exemplo, se $\rho=0, \tau=0$ e $\alpha=1$, então não há energia refletida ou transmitida e $100 \%$ da radiação incidente é absorvida. Isso é o que se chama de um corpo negro perfeito (HUDSON JUNIOR, 1969).

A Lei de Kirchhoff da radiação térmica descreve matematicamente o comportamento dos corpos com respeito ao calor irradiado. As propriedades ligadas à irradiação da energia são denotadas pelo símbolo $\varepsilon$, chamado emissividade do corpo. Essa lei prevê que $\alpha=\varepsilon$, ou, como ambas dependem do comprimento de onda, $\alpha(\lambda)=\varepsilon(\lambda)$. A equação (2), para o caso de corpos opacos $(\tau=0)$, pode ser simplificada por:

$$
\rho+\varepsilon=1
$$

No caso do corpo negro tem-se $\rho=0$ e portanto, $\varepsilon=1$.

A Lei de Planck descreve a quantidade de energia irradiada por um corpo negro em equilíbrio térmico e foi formulada em 1900 com constantes empiricamente determinadas (WYATT, 1987). Planck descreveu com precisão a radiação do corpo negro, propondo que a radiação eletromagnética era emitida em quanta. A Figura 2 mostra a emissão de radiação de um corpo negro em função do comprimento de onda, para diferentes temperaturas.

Na figura observa-se que, para temperaturas abaixo de $5000 \mathrm{~K}$, as maiores potências de radiação emitidas pelos corpos negros em equilíbrio térmico estão na zona classificada como infravermelho, o que mostra que o imageamento infravermelho é uma boa opção para visualização nessa faixa de temperaturas.

Figura 2: Potência de radiação em função do comprimento de onda.

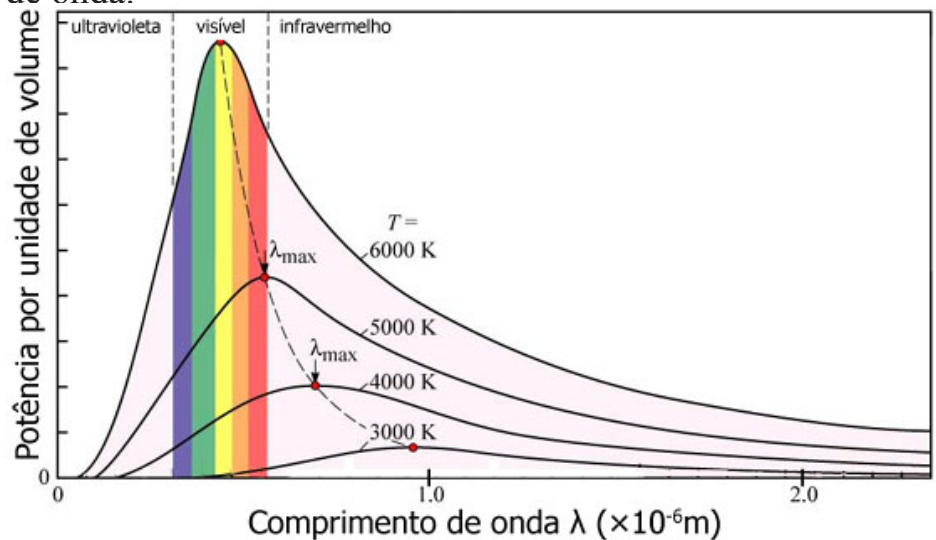

Fonte: Schappo, Brick e Barcellos (2008). 
Além disso, Planck resolveu uma imprecisão baixos comprimentos de onda, a emissão de na teoria clássica conhecida como "catástrofe do ultravioleta". Segundo a teoria clássica, para

radiação tenderia ao infinito, conforme mostrado na Figura 3.

Figura 3: Comparação entre a teoria clássica de radiação e a catástrofe do ultravioleta.

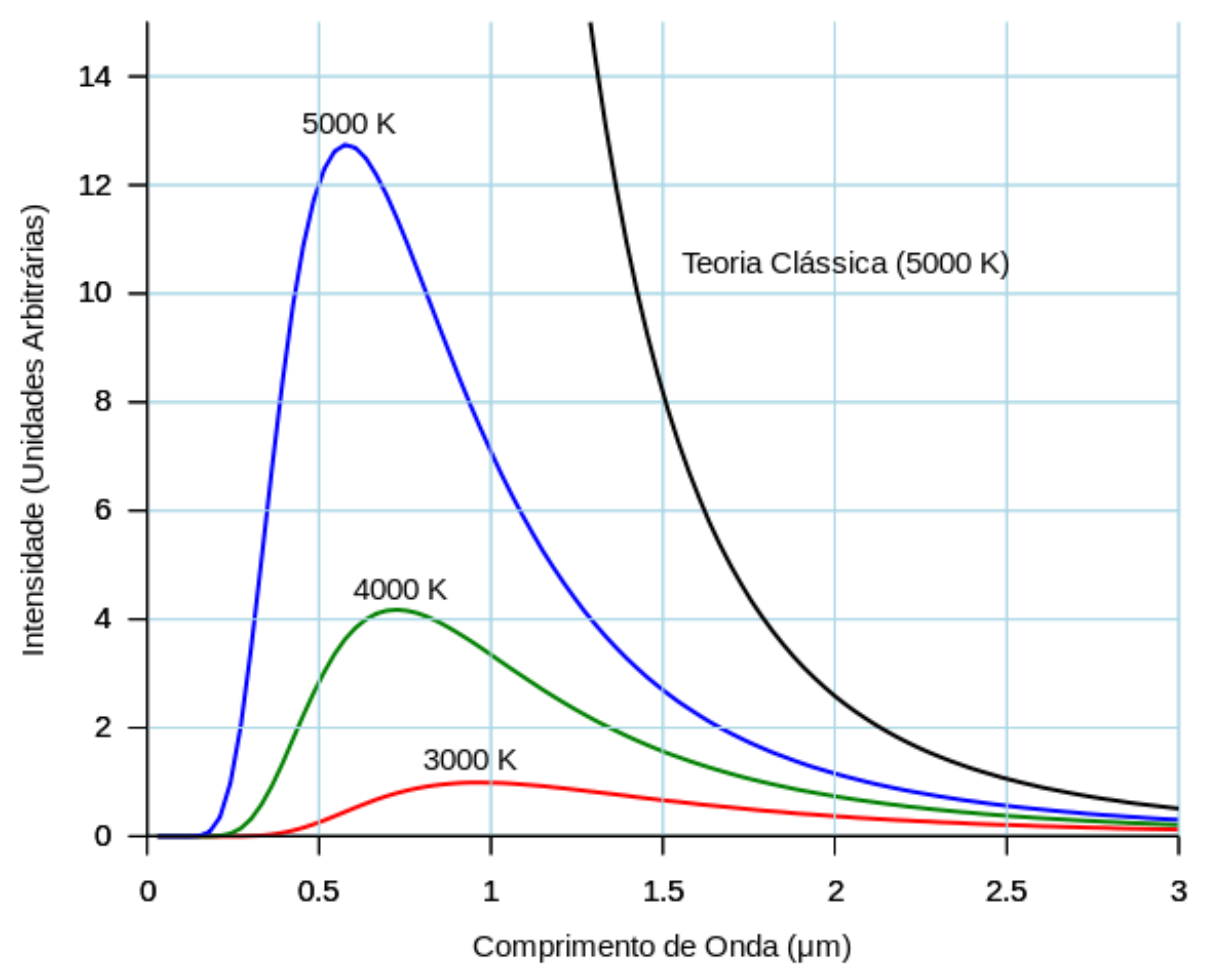

Fonte: Ponto Ciência, 2013.

Para solução, ele sugeriu que a emissão de energia eletromagnética $(u)$ era emitida em pacotes discretos de energia proporcionais à sua frequência. Esses pacotes depois foram conhecidos como fótons e tiveram papel fundamental na origem da mecânica quântica. Ainda a partir dessa formulação foi possível se determinar a constante de Planck $(h)$ usada na equação (4).

$$
u(v, T)=\frac{\mathbf{4} \pi}{c} I(v, T)=\frac{\mathbf{8} \pi \boldsymbol{h} v^{3}}{c^{3}} \frac{1}{e^{\frac{h}{k T}}-1}
$$

Onde $I$ é a radiância espectral $\left[\mathrm{Js}^{-1} \mathrm{~m}^{-2} \mathrm{sr}^{-1} \mathrm{~Hz}^{-1}\right]$; $v$ é a frequência de radiação [Hz]; $T$ é a temperatura do corpo negro [K]; $h$ é a constante de Planck $\left(6,62 \times 10^{-}\right.$ $\left.{ }^{34} \mathrm{~J} / \mathrm{Hz}\right) ; c$ é a velocidade da luz $\left(3,0 \times 10^{8} \mathrm{~m} / \mathrm{s}\right) ; e$ é o número de Euler; $k$ é a constante de Boltzmann $\left(1,38 \times 10^{-23} \mathrm{~J} / \mathrm{K}\right)$

Comprovando que a teoria dos "pacotes" era correta, Planck provou que a energia destas oscilações é limitada a múltiplos inteiros da energia fundamental $E$, e proporcional à frequência de oscilação $v$, conforme mostra a equação (5).

$$
E=\boldsymbol{h} v
$$

Ele assumiu essa quantização, cinco anos depois de Albert Einstein ter sugerido a existência de fótons para explicar o fenômeno do efeito fotoelétrico.

A partir da Lei de Planck, descobriu-se que a 
energia total irradiada por um corpo negro poderia ser calculada, e pela lei de Stefan-Boltzmann descobriu-se que ela é proporcional à quarta potência da sua temperatura em Kelvin.

$$
W=\sigma T^{4}
$$

onde $\sigma$ é a constante de Stefan-Boltzmann $\left(5,67 \times 10^{-}\right.$ $\left.{ }^{8} \mathrm{Wm}^{2} \mathrm{~K}^{4}\right)$.

As propriedades de objetos radioativos são geralmente descritas em relação a um corpo negro perfeito. Se a energia emitida a partir de um corpo negro é denotada como $W_{b b}$ e a de um objeto normal à mesma temperatura como $W_{o b j}$, a relação entre estes dois valores descreve a emissividade $(\varepsilon)$ do objeto,

$$
\varepsilon={ }^{W_{o b j}} / W_{b b}
$$

Por conseguinte, a emissividade é um número entre 0 e 1 . Quanto melhor as propriedades radioativas do objeto, mais elevada será sua emissividade. Um objeto que tem a mesma emissividade para todos os comprimentos de onda é chamado um corpo cinza.

As câmeras infravermelho atuais conseguem transformar a radiação emitida por um objeto em uma imagem. A radiação infravermelho é emitida por todos os objetos com temperaturas acima do zero absoluto e possui características semelhantes às da radiação visual como reflexão, refração e transmissão. Quanto maior a temperatura do corpo, maior será a energia irradiada.

Por esse motivo, o princípio de funcionamento de uma câmera infravermelho é semelhante ao de uma câmera para luz no comprimento de onda visível. Elas possuem uma lente que foca a radiação sobre o detector, e um conjunto de hardware eletrônico e software para processamento e amostra dos sinais e imagens gerados (Figura 4).

Portanto, a termografia permite que se faça medições de temperatura de um objeto sem contato físico. Os detectores infravermelho de câmeras modernas são compostos de uma matriz de plano focal ou Focal Plan Array (FPA) de vários materiais sensíveis a um comprimento de onda específico. A resolução da matriz de sensores pode variar de cerca de $160 \times 120$ pixels até $1024 \times 1024$ pixels. Dependendo do tamanho e resolução da uma matriz de detectores ela pode conter de 60.000 a mais de 1 milhão de detectores individuais.

Figura 4: Diagrama simplificado de uma câmera infravermelho.

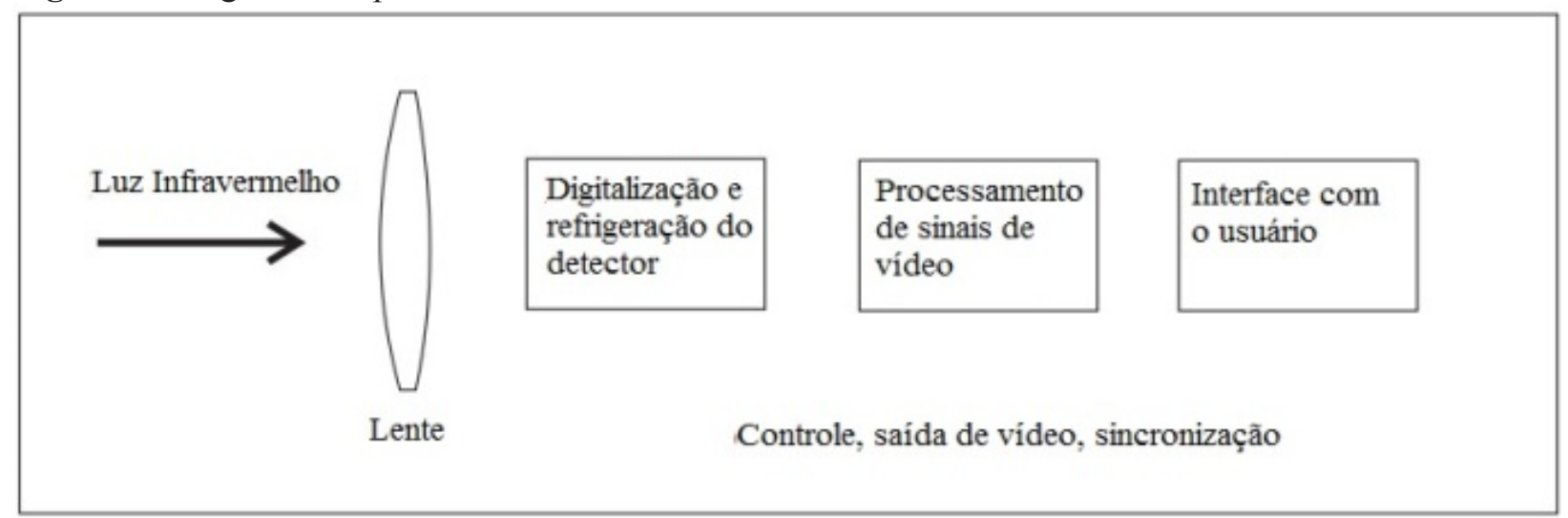

Fonte: Próprio Autor.

Além disso, muitos fabricantes de câmeras fornecem softwares que permitem que as câmeras se concentrem em áreas específicas do plano focal para medir a temperatura. As câmeras infravermelho possuem curvas de calibração que transformam a energia irradiada em temperatura na superfície do objeto. 


\section{Camada Limite}

O estudo do escoamento aerodinâmico viscoso envolve, além da própria viscosidade do fluido, efeitos de condutividade térmica do mesmo, já que ambas as propriedades influenciam de forma considerável na sua dinâmica e possuem influência mútua.

Para este estudo, pode ser feita uma analogia do fluido como sendo várias camadas sólidas deslizando uma sobre a outra, como folhas de papel (ANDERSON, 1991). Deslizando este conjunto de folhas em um objeto fixo, como uma mesa, observa-se que a velocidade aumenta nas camadas mais distantes do objeto, ou seja, a camada imediatamente adjacente à superfície tem velocidade zero e, gradualmente, cresce à medida que estas se afastam até que se atinja a velocidade nominal do escoamento. A região perto da superfície imersa no fluxo, onde pode-se observar este perfil com um gradiente de velocidade, é chamada camada limite.

Considerando um caso em que a pressão do escoamento aumenta gradualmente, haverá em um certo ponto uma região com fluxo inverso, gerando vórtices, determinando o chamado ponto de separação aerodinâmica (HOUGHTON, CARPENTER, 2004). No caso de aerofólios, a situação mais comum é a separação no ponto que determina o fim do escoamento laminar e o início do turbulento, conforme ilustrado na Figura 5.

Quando o escoamento é desacoplado, torna-se turbulento e o perfil de pressão muda drasticamente, como mostrado na mesma figura.

Figura 5: Ponto de transição e sua influência na camada limite e pressão.
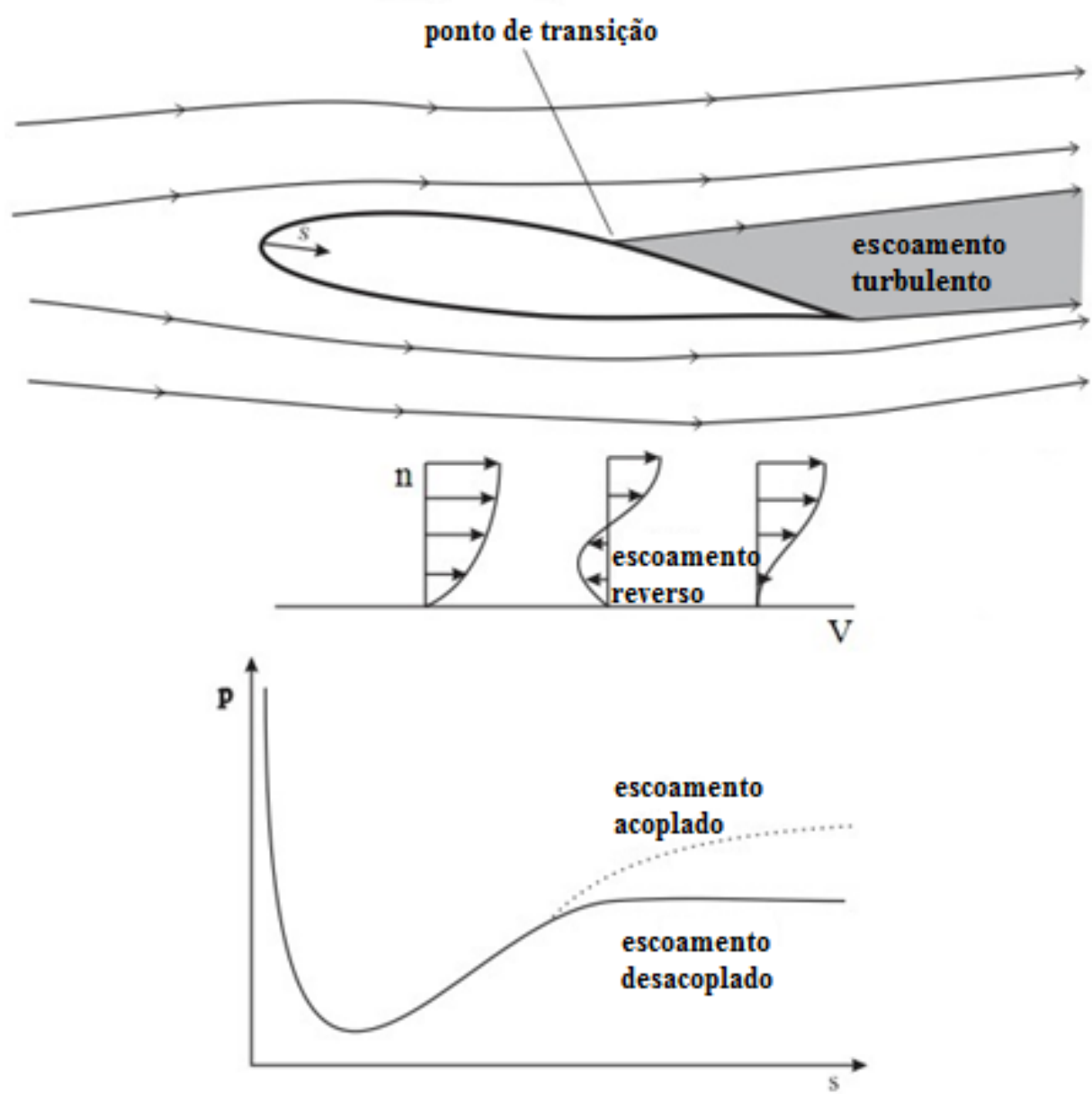

Fonte: Adaptado de Anderson (1991). 
Um perfil turbulento, por sua vez, tem algumas particularidades:

- A primeira refere-se ao gradiente de velocidade, que é consideravelmente mais elevado na região próxima à superfície aerodinâmica, conforme descrito na equação (8) e ilustrado na Figura 6.

$$
\left[\left(\frac{\partial V}{\partial n}\right)_{n=0}\right]_{\text {turbulent }}>\left[\left(\frac{\partial V}{\partial n}\right)_{n=0}\right]_{l a \min a r}
$$

Figura 6: Perfil de pressão em camada limite para escoamentos laminar e turbulento.

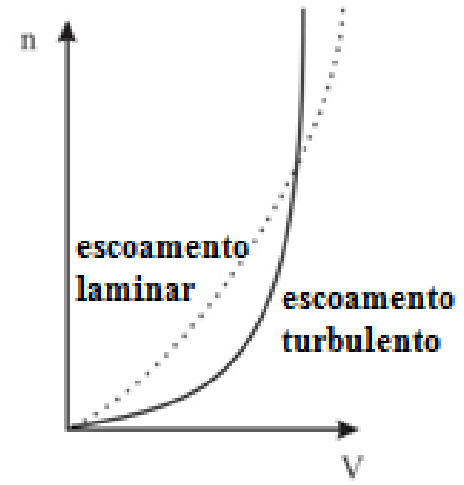

Fonte: Adaptado de Anderson (1991).

- As linhas de fluxo são mais caóticas em escoamentos turbulentos.

Figura 7: Linhas de fluxo para escoamentos laminar e turbulento.

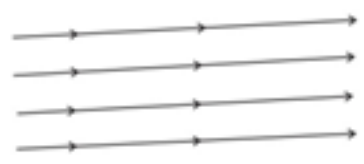

laminar

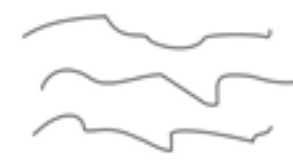

turbulento
Fonte: Adaptado de Anderson (1991)

- Devido ao seu perfil caótico, a camada turbulenta é mais espessa, uma vez que o fluxo possui maior difusividade de momento
(SCHLICHTING, GERSTEN, 2000), como mostrado na Figura 8.

Figura 8: Espessura da camada limite.
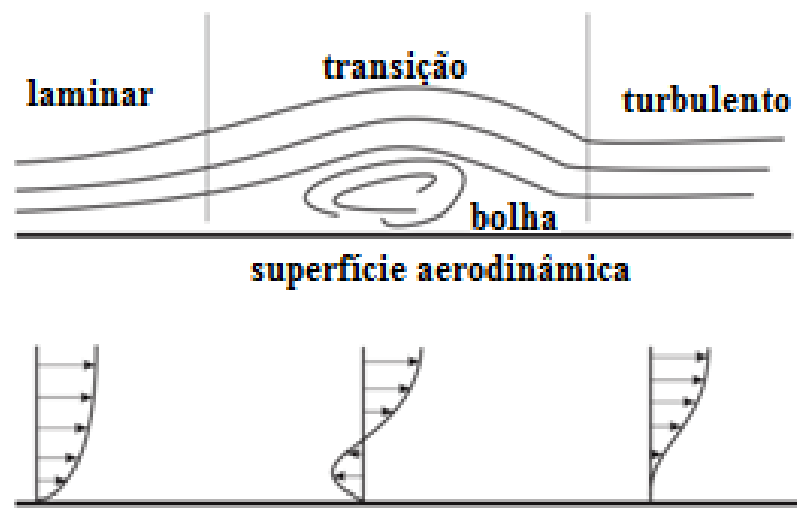

perfil da camada limite

Fonte: Adaptado de Anderson (1991).

- Em alguns casos, na transição de laminar a turbulento, pode apresentar uma "bolha" onde o fluxo não está ligado à superfície (THWAITES, 1960) como mostrado na figura 8. Esta região tem a temperatura mais baixa do que o resto do escoamento apresentado devido à diferença na troca de calor com o ambiente.

Com estas considerações, e tendo em mente a analogia feita anteriormente nesta seção, pode-se afirmar que:

1. A troca de calor da superfície com o meio num escoamento turbulento é modificada, uma vez que o atrito entre "camadas" irregulares dissipa e difunde mais energia.

2. A condução de calor entre a superfície aerodinâmica e o fluido é mais eficaz em um escoamento laminar, uma vez que o movimento ordenado das camadas facilita um maior número de moléculas em contato a superfície, aumentando a troca.

3. Na "bolha" da região de transição, a troca de calor é consideravelmente prejudicada, já que o vórtice gerado pela mesma não permite que o calor 
seja dissipado para o ambiente.

Em termos práticos, tem-se que duas situações podem ocorrer no caso de um escoamento sobre uma asa (FLIR SYSTEMS, 2012): no caso de um escoamento mais quente do que a superfície, com base na primeira conclusão, pode-se afirmar que a superfície da asa seria mais quente quando sujeita a um escoamento laminar do que no caso turbulento. No caso de um escoamento mais frio do que a superfície da asa, pode-se dizer que a sua superfície deve ser mais quente em fluxo turbulento, de acordo com a segunda indicação acima.

Ainda, é de se esperar que, caso haja uma "bolha" na região de separação, ela deve ser a região mais quente do escoamento, podendo ser facilmente detectada por sensores térmicos, tais como câmaras de imageamento de infravermelho.

\section{Metodologia}

Foram realizados ensaios em túnel de vento a fim de testar a possibilidade de visualizar a transição da camada limite com sensores térmicos e validar a metodologia para futuras aplicações em ensaios em voo.

Os testes foram realizados na Escola de Engenharia de São Carlos - USP, em um túnel de vento pequeno, de circuito aberto, utilizado para aulas práticas de aerodinâmica.

Dois perfis aerodinâmicos 2D foram testados. $\mathrm{O}$ primeiro foi um aerofólio supercrítico, já presente no laboratório e fabricado sem medidas específicas, com 100 milímetros corda e o segundo é um NACA 0012, com 150 milímetros de corda. Ambos foram pintados em preto fosco expostos a um escoamento de $28 \mathrm{~m} / \mathrm{s}$, com cerca de $27^{\circ} \mathrm{C}$ de temperatura e $5^{\circ}$ de ângulo de ataque.

Existem duas técnicas clássicas para aumentar o contraste térmico durante este tipo de teste. A primeira é a chamada "técnica passiva", onde o fluxo de ar é aquecido antes de atingir a superfície de sustentação, e a segunda, chamada "técnica activa", em que a superfície do perfil aerodinâmico é aquecida antes dos testes (LAMAR, 2001; MALERBA et al., 2008).

Devido às configurações de túnel de vento, a técnica ativa foi escolhida, de forma a aumentar o contraste e obter resultados mais satisfatórios, substituindo a primeira tentativa (sem aquecimento) que não possibilitou bom contraste. $\mathrm{O}$ aquecimento da superfície foi feito em uma estufa com lâmpadas elétricas, utilizado em atividades de laminação com fibra de vidro.

A câmera infravermelho utilizada foi um modelo FLIR P620, e suas imagens foram analisadas usando o software FLIR QuickReport SP2 v1.2, que tornou possível uma análise completa, gerando medidas de temperatura e alterações de alguns parâmetros de visualização, como a faixa de temperatura e escalas para a imagem.

Após os testes com visualização térmica, ambos os aerofólios foram testados, nas mesmas condições anteriores com "flow-viz" (visualização do fluxo aerodinâmico com um fluido viscoso aplicado na superfície do aerofólio), a fim de comparar os resultados e validar o ensaio.

Os resultados do aerofólio NACA 0012 foram ainda comparados com os dados apresentados na bibliografia, de forma a se confrontar os métodos e o resultado esperado.

\section{Resultados}

\section{Perfil supercrítico}

Um aerofólio assimétrico de perfil supercrítico foi montado no túnel de vento e o escoamento foi iniciado nas condições descritas no item anterior. A figura 9 mostra o aerofólio em uma imagem infravermelho antes de iniciar o escoamento. 
Figura 9: Imagem térmica do perfil supercrítico antes do escoamento.

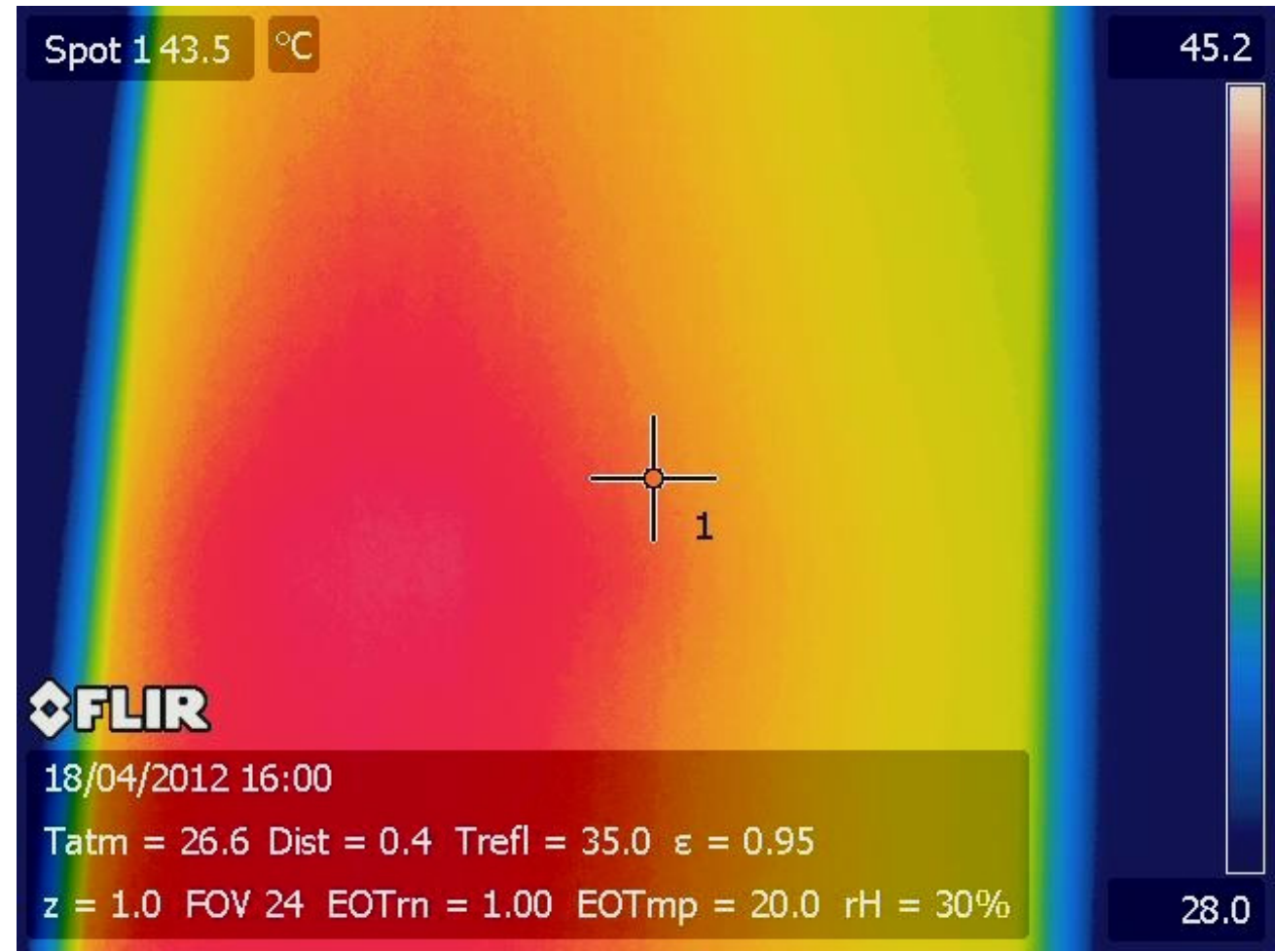

Fonte: Próprio autor.

Esta figura apresenta uma vista de topo do perfil onde se pode identificar a distribuição de temperatura ao longo do mesmo.

Nota-se que antes do início do experimento a temperatura não se mostra uniforme ao longo do perfil. O motivo disso é a troca de calor com o ambiente no período em que o perfil foi retirado da estufa e montado na câmara de ensaios do túnel

Observa-se na figura 10 que a temperatura diminui imediatamente após o início do escoamento, devido ao aumento do coeficiente de troca de calor por convecção, com o ar em movimento em contato com a superfície. Notase também a formação da bolha de separação laminar com reacoplamento na forma turbulenta mais adiante. $\mathrm{Na}$ região do bordo de ataque a temperatura diminui, devido ao alto coeficiente de troca de calor por convecção graças à pequena espessura da camada limite. Ao longo da superfície a espessura da camada limite aumenta e, consequentemente, o coeficiente de transferência de calor por convecção diminui. Como consequência, observa-se o gradiente de temperatura obtido no teste, onde tais variações entre o bordo de ataque e a bolha de separação é de cerca de $4{ }^{\circ} \mathrm{C}$.

Pode-se observar também que na região central da envergadura, a bolha apresenta tamanho maior. Tal fato foi associado à camada limite das paredes do túnel, que fizeram com que a troca de calor tivesse pequenas diferenças ao longo da envergadura da asa.

Comparando a figura 10 com os resultados obtidos pelo método "flow-viz" mostrado na figura 11, há uma semelhança clara no comportamento do escoamento. Em ambos os casos, há a formação de bolha de separação laminar e o reacoplamento em posições bem definidas ao longo da superfície. 
Figura 10: Aerofólio supercrítico exposto ao escoamento $\left(5^{\circ}\right.$ de ângulo de ataque).

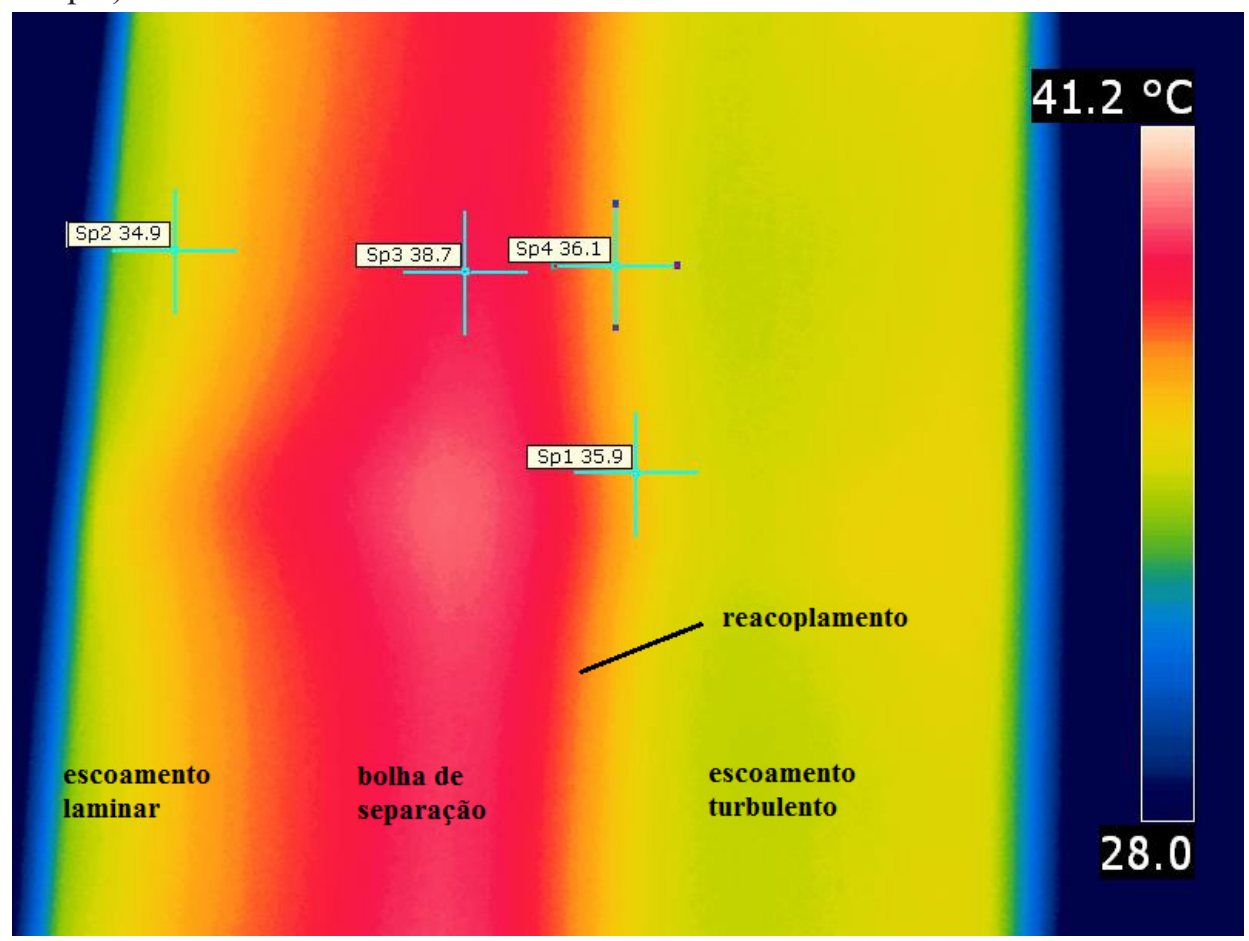

Fonte: Próprio autor.

Figura 11: Aerofólio supercrítico exposto ao escoamento $\left(5^{\circ}\right.$ de ângulo de ataque).

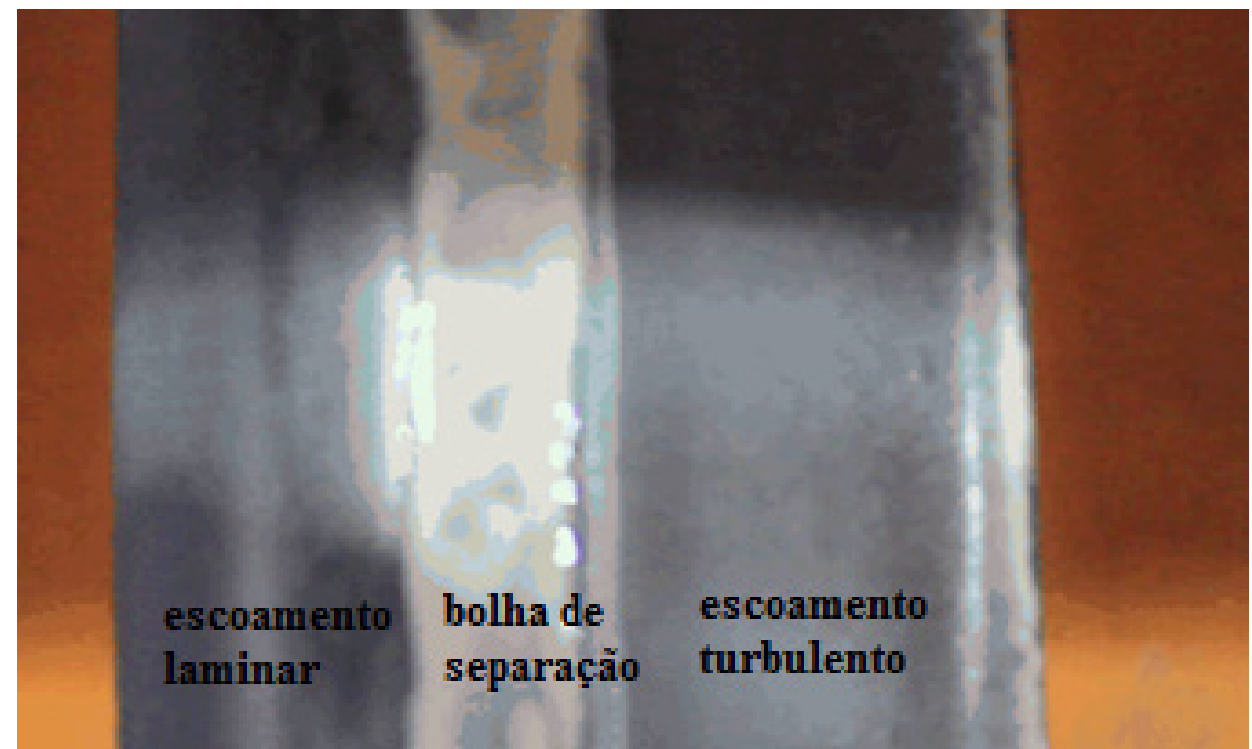

Fonte: Próprio autor.

$\mathrm{O}$ ajuste de câmera infravermelho é um dos fatores importantes para bons resultados. Houve alguma dificuldade em focar a câmera por causa da curta distância entre a janela do túnel e o modelo (cerca de $300 \mathrm{~mm}$ ). A emissividade assumida foi de 0,98 e a temperatura refletida foi assumida igual 
à temperatura atmosférica. Estes parâmetros são configuráveis após o teste usando o software FLIR QuickReport v1.2 SP2. Através da manipulação das imagens pode ser visto que a variação de temperatura entre o bordo de ataque do perfil laminar e a bolha é maior do que $4{ }^{\circ} \mathrm{C}$ durante as condições de teste.

Outro fato relevante é que o contraste de calor necessário para obter imagens de infravermelho foi obtido por aquecimento dos modelos uniformemente antes do teste no túnel de vento. Após o início do escoamento, o perfil de temperaturas observado na figura 11 durou um determinado período de tempo (cerca de 5 minutos), após o qual o contraste da imagem infravermelho tornou-se menos claro, indicando que os gradientes térmicos diminuíram dificultando, assim, a visualização.

\section{Perfil NACA 0012}

O mesmo procedimento foi repetido para um aerofólio NACA 0012, com a vantagem de esse ser um perfil extensivamente apresentado na literatura.

Próximo ao bordo de ataque do aerofólio foi posicionado um gerador de vórtice de modo a se estabelecer um parâmetro de avaliação para as características do escoamento no perfil. Além disso, ao longo da corda perfil, tiras de alumínio foram colocadas para servir como marcas nas imagens infravermelho e para ajudar na análise dos resultados observados. A primeira fita de alumínio iniciou-se no bordo de ataque e terminou a $25 \%$ da corda e a segunda começou em $50 \%$ da corda e seguiu até $75 \%$ da mesma, como mostrado na figura 12 .

Figura 12: Imagem térmica do perfil NACA 0012 antes do escoamento.

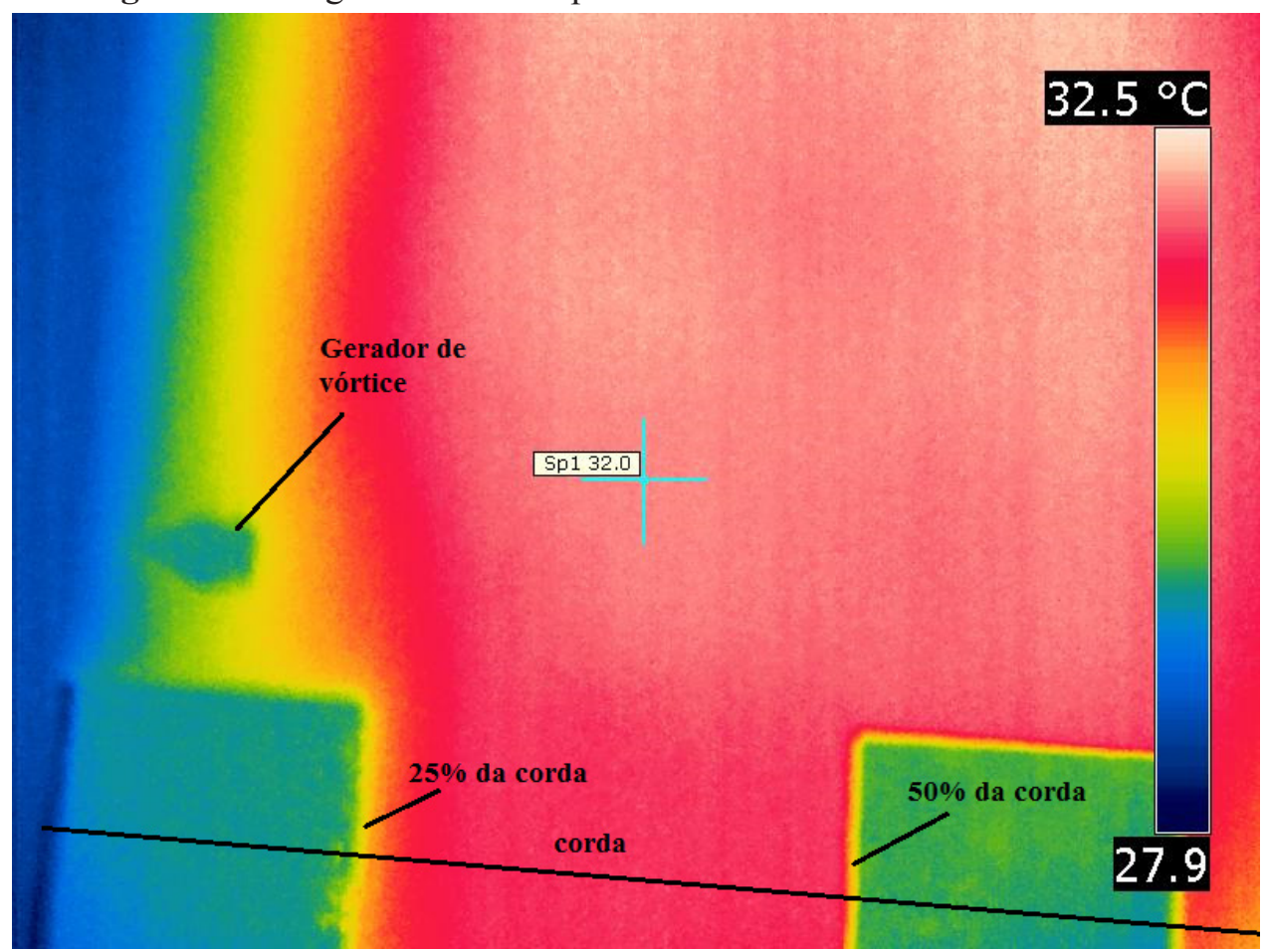

Fonte: Próprio autor.

$\mathrm{Na}$ figura 13, duas informações importantes podem ser obtidas. A primeira é o comportamento do escoamento através do gerador de vórtice, em que se pode observar uma diminuição de temperatura gerada nesta região, o que demonstra a eficácia do presente método em observações aerodinâmicas deste e outros tipos. 
Figura 13: Imagem térmica do perfil NACA 0012 sujeito ao escoamento $\left(5^{\circ} \mathrm{de}\right.$ ângulo de ataque).

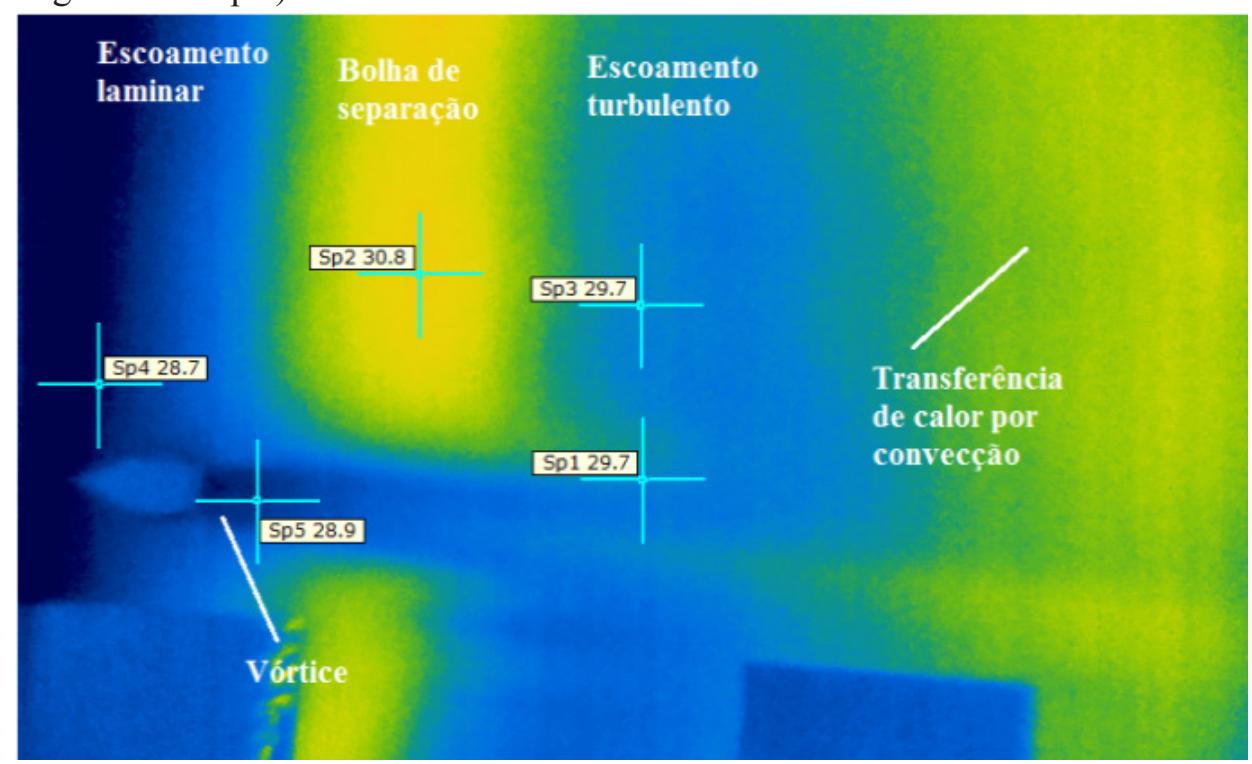

Fonte: Próprio autor.

A segunda consideração é a transição por um aumento local de temperatura. Observandoaerodinâmica de laminar a turbulento. Comparando se a figura 14, pode-se comparar o presente método a figura 13 com a figura 10, o mesmo padrão pode ser com o método 'flow-viz', obtendo-se resultados observado, com a bolha de separação determinada bastante semelhantes.

Figura 14: Aerofólio NACA 0012 exposto ao escoamento $\left(5^{\circ}\right.$ de ângulo de ataque).

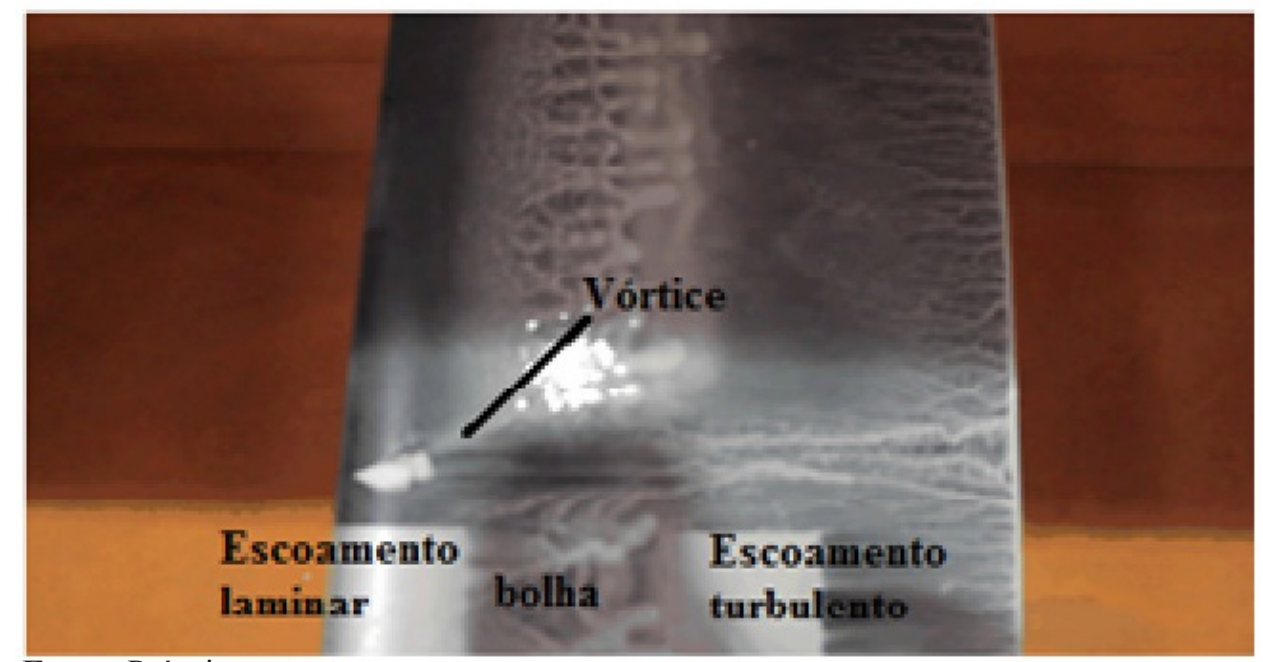

Fonte: Próprio autor.

As imagens obtidas estão relacionados com os dados apresentados na bibliografia (GREGORY, O’REILLY, 1973), que investigaram o aerofólio
NACA 0012 e mostra que, com 5 graus de ângulo de ataque, a bolha separação começa a cerca de $15 \%$ da corda do aerofólio. A mesma 
informação é apresentada como um resultado de simulação por este aerofólio usando software XFLR5, mostrando na figura 15 , em que uma brusca redução do coeficiente de pressão em torno de $15 \%$ da corda pode ser observada, o que caracteriza o início da transição.

Figura 15: Resultado de simulação pelo software XFLR5 do aerofólio NACA 0012 com $5^{\circ}$ de ângulo de ataque.

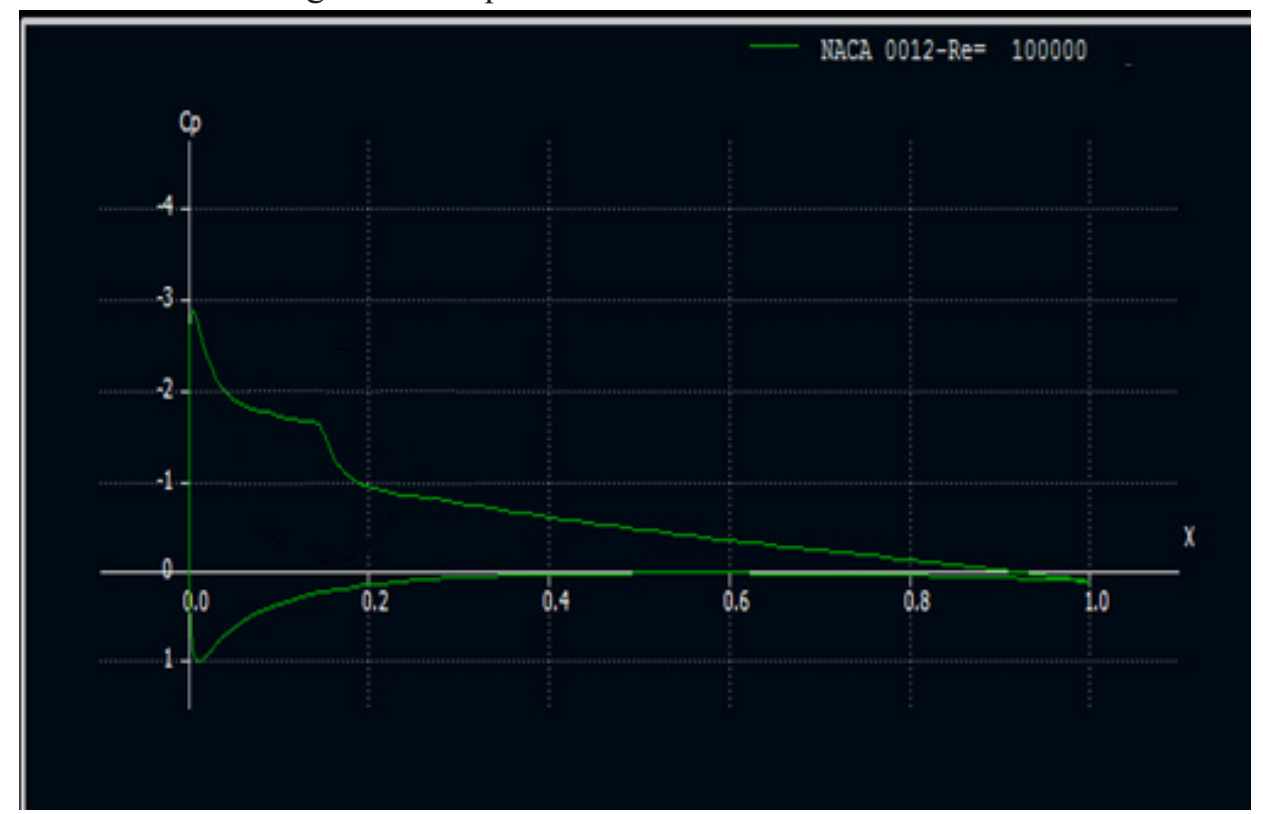

Fonte: Próprio autor.

\section{Conclusões}

Resultados preliminares mostram que a observação de transição da camada limite com câmera infravermelho fornece resultados satisfatórios e consistentes em comparação com outro método de visualização. Os testes de túnel de vento mostraram que o método tem potencial para uso em ensaios mais complexos e pode ser vantajoso para vários tipos de aplicação, como ensaios de voo ou testes em túnel de vento, mas algumas dificuldades adicionais devem ser superados, tais como:

- Tratamento da superfície a ser investigada com tinta de alta emissividade (de preferência preto fosco, outras cores devem ser investigados);

- Posicionamento da câmera por um pod ou instalada em uma aeronave-paquera ou na câmara do túnel de vento;

- Possíveis efeitos térmicos indesejáveis, tais como os gerados por tanques de combustível ou de outras estruturas e suportes instalados sob a superfície de interesse.

Outras dificuldades ainda precisam ser melhor pesquisadas como a melhor configuração para análise dos dados da câmera via software. Tais problemas estão em estudo e devem ser tratados antes de seu uso em aeronave real.

Outros testes devem ser realizados no futuro, antes de testes de voo:

- Testes em túnel de vento com outras pinturas 
de cores e tintas aeronáuticas;

- Testes em túnel de vento com câmeras de maior sensibilidade e alta amostragem;

- Testes em túnel de vento para verificar a possibilidade de visualização de separação aerodinâmica com ângulos de ataque mais elevados;

- Utilização da técnica em túneis de vento maiores para alcançar números de Reynolds mais elevados;

- Testes em túnel de vento para verificar detecção dos efeitos da esteira de turbulência gerados por superfícies aerodinâmicas e turbinas a jato usando termografia.

\section{Referências}

ANDERSON, J. F. Fundamentals of aerodynamics. 2nd ed. New York: McGraw-Hill, 1991.

BANKS, D. W. Visualization of in-flight flow phenomena using infrared thermography. NASA TM-2000-209027, 2000.

CLIFTON, J. M. Introduction to avionics flight test. London: AGARDograph, 1996. v. 15.

DEHNE, T.; BOSBACH, J.; HEIDER, A. Dynamics of arcraft cabin ventilation by in-flight infrared thermography. In: INTERNATIONAL CONFERENCE ONQUANTITATIVE INFRARED THERMOGRAPHY - QIRT, 11., 2012, Naples, Italy, 2012.

FISHER, D.; HORSTMANN, K. H.; RIEDEL, H. Flight test measurement techniques for laminar flow. London: AGARDograph, 2003. v. 23.

FLIR SYSTEMS. The ultimate infrared handbook for $R \& D$ professionals. Sweden: FLIR Systems AB, 2012.

GREGORY, N.; O'REILLY, C. L. Low-speed aerodynamic characteristics of NACA 0012 aerofoil section, including the effects of upper-surface roughness simulating hoar frost. Aeronautical Research Council, No RC 3726, 1973.
HOLST, G. C. Electro-optical imaging system performance. 5th ed. Winter Park, FL: JCD Publishing, 2003.

HOUGHTON, E. L.; CARPENTER, P. W. Aerodynamics for engineering students. 5th ed. Burlington, MA: Elsevier Butterworth-Heinemann, 2004.

HUDSON JUNIOR, R. D. Infrared system engineering. Gainesville, FL: John Wiley \& Sons, 1969.

LAMAR, J. E. Flow visualization techniques used at high speed by configuration aerodynamics WindTunnel-Test Team, NASA report, No TM- 2001210848, 2001.

MALERBA, M. et al. A boundary layer inspection on a wing profile through high resolution thermography and numerical methods. WSEAS Transactions on Fluid Mechanics, Perugia, Italy, v. 3, p. 18-28, 2008.

McSHEA, R. E. Test and evaluation of aircraft avionics and weapon systems. Raleigh, NC: SciTech Publishing, Inc., 2010.

PION: Ligado na Física. Espectro eletromagnético. 2012. Disponível em: <www.sbfisica.org.br/v1/ novopion/index.php/publicacoes/imagens/130espectro-eletromagnetico $>$. Acesso em: 22 jan. 2014.

PONTO CIÊNCIA. Emissão de um corpo negro. 2013. Disponível em: <http://pontociencia.org.br/ galeria/?content $\% 2$ FFisica $\% 2$ FFisica+Moderna $\% 2$ Fcorpo-negro.jpg>. Acesso em: 22 jan. 2014.

SCHAPPO, M. G.;BRICK, E. M.; BARCELLOS,A. B. Lâmpadas incandescentes. 2008. Disponível em: $<$ http://www.ced.ufsc.br/men5185/trabalhos/63 lampadas/incand/funciona04.htm>. Acesso em: $2 \overline{2}$ jan. 2014.

SCHLICHTING, H.; GERSTEN, K. Boundary layer theory. $8^{\text {th }}$ ed. Berlin: Springer, 2000.

THWAITES, B. Incompressible aerodynamics. Oxford: Dover, 1960. 
WYATT, C.L. Radiometric system design. New York: Macmillan Publishing Co., 1987.

ZUCCHER, S. et al. The role of infrared thermogaphy in the study of crossflow instability at $M=2.4$. In: INTERNATIONAL SYMPOSIUM ON FLUID CONTROL, MEASUREMENT AND VISUALIZATION, 7., 2003, Sorrento. Proceedings... Sorrento, Italy, 2003. 
